\title{
Choroidal Thickness in Patients with Diabetic Macular Edema and its Correlation to Macular Thickness and Vision
}

\author{
Mona N. Mansour, Hanan S. Hegazy
}

Department of Ophthalmology, Faculty of Medicine (for Girls), Al-Azhar University, Cairo, Egypt.

Corresponding author: Mona N. Mansour, Tel: +20 101698 3770; fax: +20 2 22638357,e-mail: mansour04@hotmail.com

\begin{abstract}
Purpose: To investigate the relationship between subfoveal choroidal thickness, central macular thickness, visual acuity and the presence of diabetic macular edema (DME) using spectral domain optical coherence tomography (SD-OCT).

Methods:A prospective, nonrandomized case control study of 124 eyes was included in the study and divided into three groups: Group I: 56 eyes having NPDR without macular edema, Group II: 27 eyes having NPDR with DME, Group III: 41 eyes of normal healthy subjects. Central macular thickness (CMT) and subfoveal choroidal thickness (SFCT) were measured on the OCT images and statistically compared. Pearson correlation analysis used to evaluate the relationships between choroidal thickness, macular thickness and visual acuity.

Results: A total of 124 eyes of 76 patients; mean age $52.56 \pm 8$.91years were included in the study. Mean bestcorrected visual acuity LogMAR was better in the control group $(0.20 \pm 0.40)$ than diabetic groups $(0.42 \pm 0.40$ in group I, $0.80 \pm 0.45$ in group II) and the difference was significant among the groups $(P=0.01)$. Mean CMT was thicker in DME group $(374.63 \mu \mathrm{m} \pm 105.43)$ than the other 2 groups $(246.66 \pm 24.85 \mu \mathrm{m}$ in group I, 227.05 $\mu \mathrm{m} \pm 21.97$ in group III) and the difference was significant among the groups $(P=0.00)$. Mean SFCT was thinner in DME group $(234.93 \mu \mathrm{m} \pm 42.68)$ than $\mathrm{d}$ the other 2 groups $(246.41 \mu \mathrm{m} \pm 44.37$ in group I, $250.20 \mu \mathrm{m}$ \pm 53.10 in group III) but the difference was not statistically significant $(P=0.41)$. A significant correlation was found between LogMAR and CMT in group II $(r=0.391, P=0.044)$ indicating that the vision may decrease as the central macular thickness increases in diabetic groups. A weak inverse correlation was found between LogMAR and SFCT in all groups indicating that the vision may decrease as the choroidal thickness decrease. A weak negative correlation was found between CMT and SFCT in diabetic groups $(r=-0.142$ in group I, $r=-0.152$ in group II) indicating that the choroidal thickness may decrease as the central retinal thickness increases in diabetic groups.
\end{abstract}

Conclusion: In diabetic eyes, there is an overall thinning of the choroid. SFCT is directly related to vision and macular thickness.

Keywords: choroidal thickness; diabetic retinopathy; diabetic choroidopathy.

\section{INTRODUCTION}

Diabetic retinopathy (DR) is the leading cause of vision loss of working-age adults and diabetic macular edema (DME) is the most frequent cause of vision loss related to diabetes ${ }^{(1)}$. Since choroidal vasculature provides oxygen and nutrients to the outer retina and is responsible for maintaining the highly metabolically active photoreceptor cells. Therefore, evaluation of the structural changes in the choroid might be very insightful to determine the pathogenesis of progression of the macular changes in diabetic eyes ${ }^{(2)}$.

The in vivo structure of the choroid is incompletely visualized with traditional imaging modalities as indocyanine green angiography, ultrasonography. Use of new OCT modalities, including enhanced depth imaging OCT (EDI-OCT) and swept-source OCT, have led to increased visualization of the choroidal anatomy ${ }^{(3)}$. Several studies on Choroidal thickness in eyes with DME revealed different results; choroidal thickening (4), thinning ${ }^{(5)}$, and no change ${ }^{(6)}$.

In this study, using EDI-OCT, we investigated the subfoveal choroidal thickness (SFCT) in eyes with diabetic retinopathy and macular edema (NPDR + DME) and compared the measured values with those of NPDR without DME and of healthy normal subjects, as well as its correlation with bestcorrected visual acuity (BCVA) and central macular thickness (CMT).

\section{PATIENTS AND METHODS}

A prospective, nonrandomized, case control study was performed on 124 eyes of 76 patients who attended to our clinic at Al-Zahraa University Hospital in Cairo, Egypt, between December 2015 and August 2017. The study adhered to the tenets of the Declaration of Helsinki and was approved by the ethical board of Al-Azhar university and informed written consent was taken from each participant in the study.

The 124 eyes were divided into three groups:

Group I: 56 eyes of diabetic patients having NPDR without macular edema.

Group II: 27 eyes of diabetic patients having NPDR with macular edema (DME).

Group III: 41 eyes of normal healthy subjects.

For inclusion in the diabetic groups, participants having nonproliferative diabetic retinopathy (NPDR), patients with proliferative 
diabetic retinopathy were excluded. The duration of diabetes and recent control of diabetes were noted. The control group are non-diabetics, based on history and laboratory investigations and absence of retinal or choroidal pathology on fundus examination.

Eyes with a retinal disease other than DR, glaucoma, ocular trauma, media opacity, high refractive error and axial length greater than $26 \mathrm{~mm}$ were excluded. Eyes that had undergone cataract surgery, panretinal photocoagulation or treatment with anti-vascular endothelial growth factor (VEGF) therapy in the last 6 months were also excluded.

All participants underwent a comprehensive ophthalmic examination including visual acuity testing, slit-lamp biomicroscopy, intraocular pressure (IOP) measurement, and dilated fundoscopic examination.

OCT scanning with a high-speed spectraldomain OCT machine (Spectralis; Heidelberg Engineering $\mathrm{GmbH}$, Heidelberg, Germany) done to measure the central macular and subfoveal choroidal thickness. Measurements was performed at fixed time of the day ( 2 p.m. to avoid circadian rhythm of the choroidal thickness) using the built-in seven-line raster scan protocol. Images were averaged from 100 frames for the purpose of noise reduction. All study eyes were dilated with mydriatic eye drops before image acquisition. Patients were instructed to fixate on the intrinsic fixation target during the whole process of OCT scanning. Measurements were made

manually using the caliber tool in the OCT Heidelberg software. The CMT was defined as the distance between the ILM and RPE at the foveal center, whereas subfoveal choroidal thickness (SFCT) was the distance from outer boundary of the RPE till end of choroid (the sclerochoroidal interface) at the foveal center (Figures1, 2, 3).

Statistical analysis was performed using Statistical Package for the Social Sciences software (SPSS 11.01) with A p-value of less than 0.05 indicated statistical significance. Comparison between groups was analyzed by using one-way ANOVA. Correlation between BCVA, CMT, and SFCT in each group was analyzed using the Pearson's correlation coefficient 
Mona N. Mansour and Hanan S. Hegazy
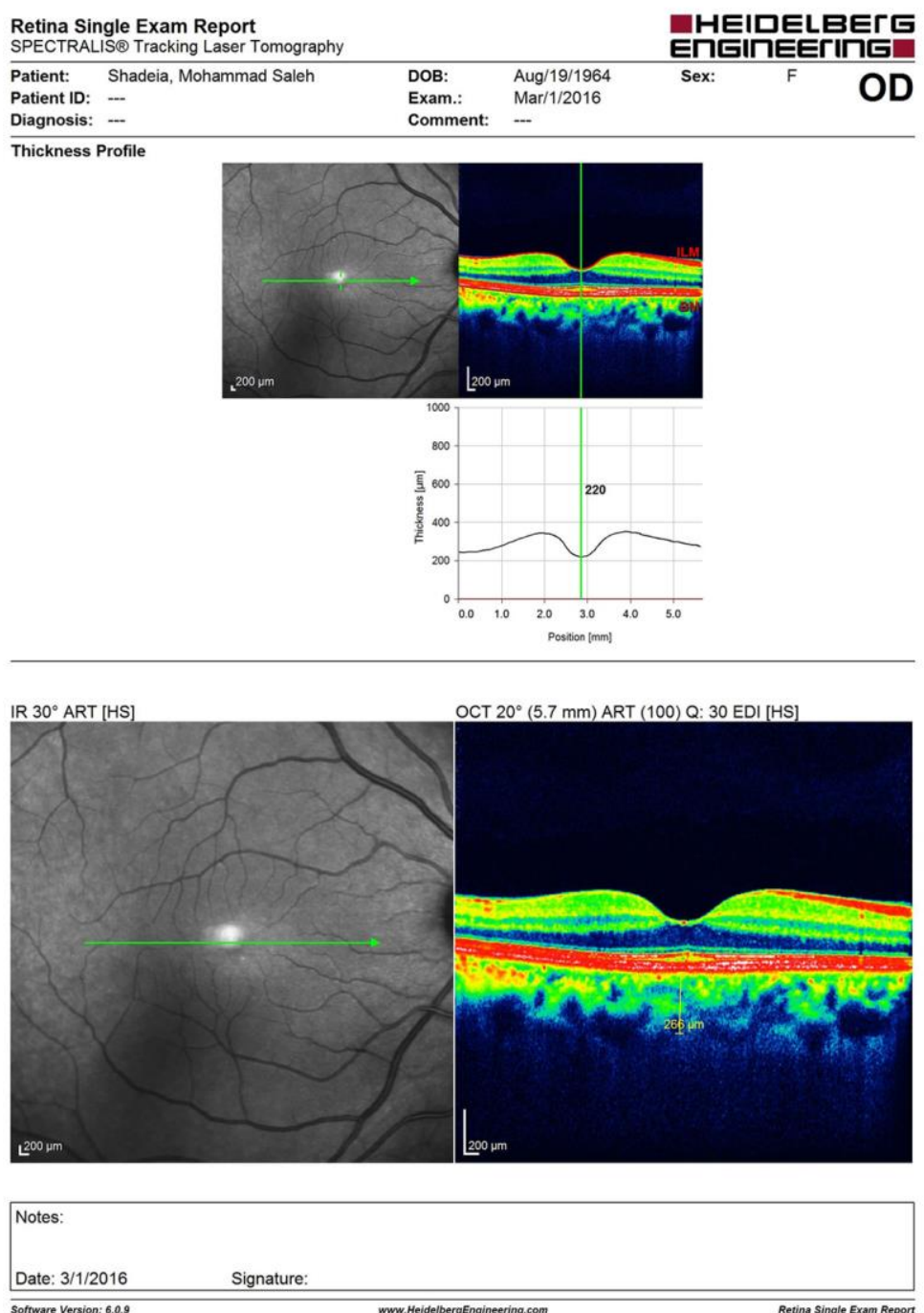

Figure 1. Female 52y old with a normal macula; CMT $220 \mu \mathrm{m}$ and SFCT $266 \mu \mathrm{m}$. 
Choroidal Thickness in Patients...
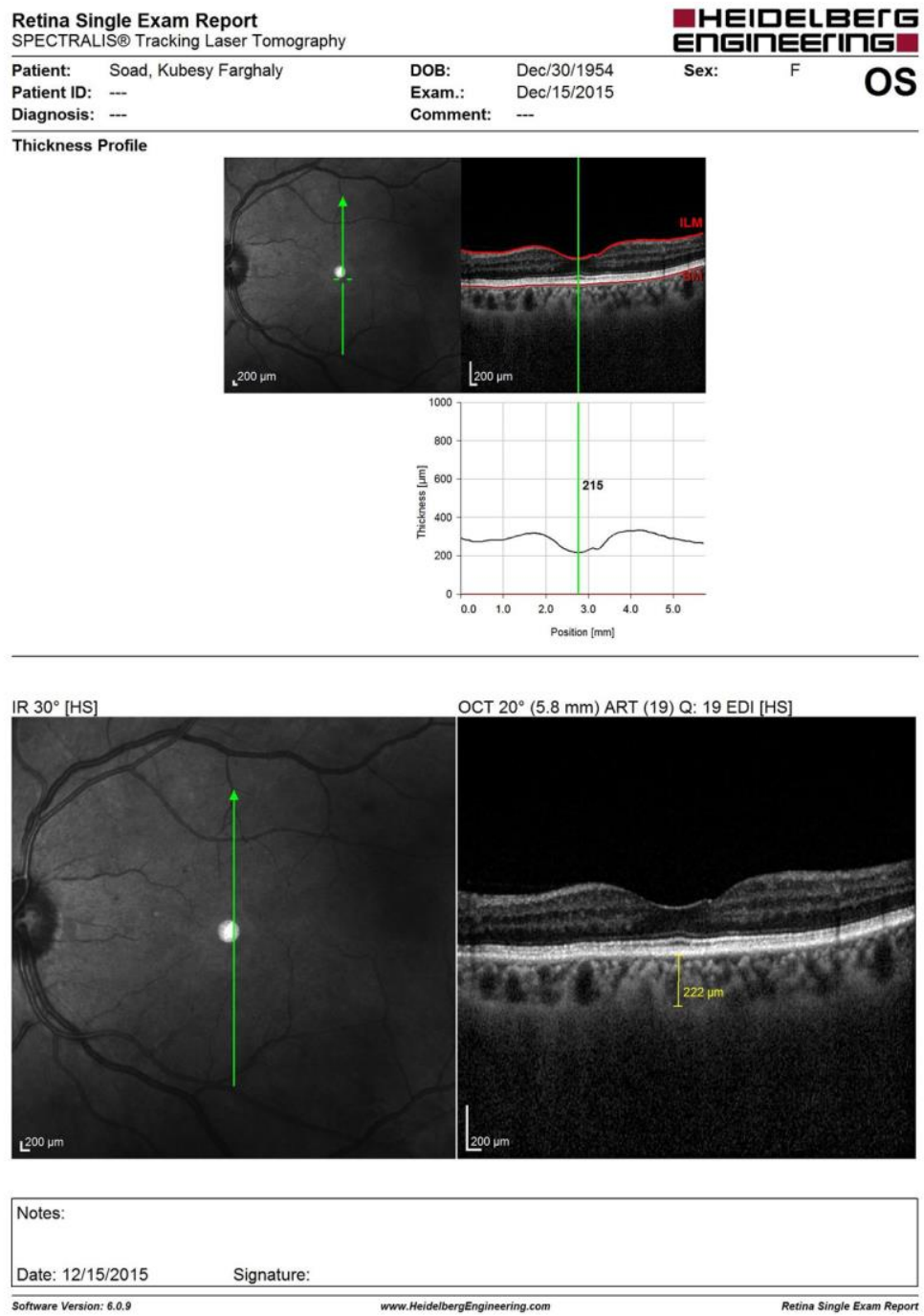

Figure 2. Female 61y. old, NPDR without DME; CMT $215 \mu \mathrm{m}$ and SFCT $222 \mu \mathrm{m}$. 

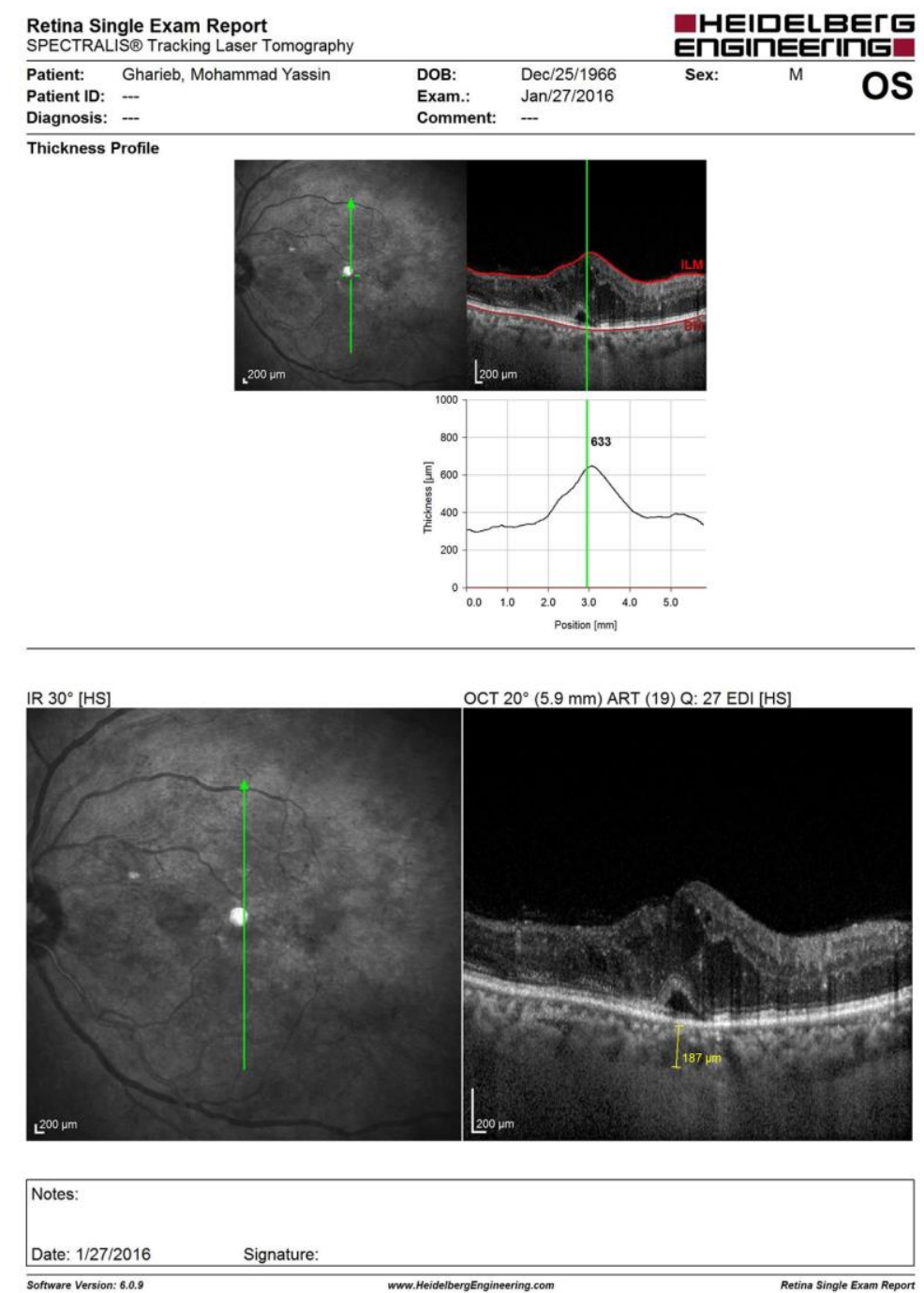

Figure 3. Male 50y. old with a NPDR+ DME (CME, SRF); CMT $633 \mu \mathrm{m}$ and SFCT $187 \mu \mathrm{m}$.

\section{RESULTS}

This prospective study included 124 eyes of 76 patients, 83 of them have NPDR, 41 normal eyes. A total of 56 eyes had NPDR (group I), 27 eyes had NPDR+ DME (group II), and 41 eyes as controls (group III). The mean

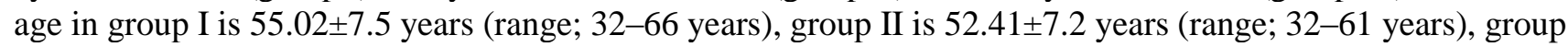
III is $49.29 \pm 10.6$ years (range; $32-68$ years). The duration of diabetes in group I is $14.79 \pm 5.58$, group II is 14.65 \pm 5.35 . Comparisons between our groups are summarized in Table 1 .

Table 1. BCVA, CMT, and SFCT in the Three Groups of Diabetic Patients and Controls.

\begin{tabular}{|l|l|l|l|l|}
\hline & group I & group II & group III & $P$ value \\
\hline BCVA (Log MAR) & $0.42 \pm 0.40$ & $0.80 \pm 0.45$ & $0.20 \pm 0.40$ & 0.01 \\
\hline CMT & $246.66 \pm 24.85$ & $374.63 \pm 105.43$ & $227.05 \pm 21.97$ & 0.00 \\
\hline SFCT & $246.41 \pm 44.37$ & $234.93 \pm 42.68$ & $250.20 \pm 53.10$ & 0.413 \\
\hline
\end{tabular}

BCVA; best-corrected visual acuity, CMT; central macular thickness, SFCT; Subfoveal choroidal thickness The mean BCVA was better in the control group (group III), (approximately 20/32; $0.20 \operatorname{logMAR}$ ), than the diabetic groups (approximately 20/50; 0.42 and 20/125; $0.80 \operatorname{logMAR}$ ). The difference was statically significant among the groups $(P=0.01)$. The CMT which was thickest in group II $(374.63 \mu \mathrm{m} \pm 105.43)$, the difference was 
statically significant among the groups $(p=0.00)$. The SFCT which was thinnest in group II $(234.93 \mu \mathrm{m} \pm 42.68)$, the difference was not statically significant among the groups $(P=0.413)$.

Table 2. The relationship between choroidal thickness, vision and foveal thickness.

\begin{tabular}{|l|l|l|l|l|l|l|}
\hline & \multicolumn{2}{|l|}{$\begin{array}{l}\text { Correlation between Log } \\
\text { MAR and CMT }\end{array}$} & \multicolumn{2}{l|}{$\begin{array}{l}\text { Correlation between Log } \\
\text { MAR and SFCT }\end{array}$} & \multicolumn{2}{l|}{$\begin{array}{l}\text { Correlation between CMT } \\
\text { and SFCT }\end{array}$} \\
\cline { 2 - 7 } & \multicolumn{1}{|c|}{$r$} & \multicolumn{1}{|c|}{$P$} & $r$ & $P$ & $r$ & $P$ \\
\hline Group I & -0.284 & 0.151 & -0.215 & 0.177 & -0.142 & 0.297 \\
\hline Group II & 0.391 & 0.044 & -0.286 & 0.148 & -0.152 & 0.248 \\
\hline Group III & -0.276 & 0.102 & -0.188 & 0.174 & 0.125 & 0.436 \\
\hline
\end{tabular}

Log MAR; Minimum angle of resolution of visual acuity, CMT; central macular thickness, SFCT; Subfoveal choroidal thickness

There was a statistically significant correlation between the $\log$ MAR and CMT in the DME group, but not in the other 2 groups, indicating that the visual acuity may decrease as the CMT increases in this group. The $r$ and $P$ values are shown in Table 2 . SFCT is inversely correlated to Log MAR in all groups, suggesting that if choroidal thickness is reduced, the vision will be affected. (Table 2)

There was a weak negative correlation between CMT and SFCT in the diabetic groups but not in the control subjects (Table 2). suggesting that the choroidal thickness may decrease as the CMT increases in the diabetic groups.

\section{DISCUSSION}

In our study, the BCVA was better in the control group than diabetic groups (group II, group III) and the difference was statistically significant among the groups $(P=0.01)$. Also previous studies reported a significantly better vision by comparing the healthy control group and the group of DME ${ }^{(7-9)}$.

In our study, the CMT of patients with DME (group II) was thicker than that of other 2 groups and the difference was statistically significant among the groups $(P=0.00)$, this consistent with other studies (7-12) that reported a statistically significant increase in CMT in DME cases compared to healthy control cases. There was a statistically significant correlation between the $\log$ MAR and CMT in the DME group (group II), but not in the other 2 groups, indicating that the visual acuity may decrease as the CMT increases in this group. These findings were similar to those found in other studies ${ }^{(7-10)}$.

In our study, the SFCT of patients with DME (group II) was thinner than that of other 2 groups but the difference was not statistically significant $(P=0.41)$. Other studies reported that the presence of diabetic macular edema is associated with a significant decrease in the choroidal thickness compared with normal subjects $(2,5,9,11,13-16)$. Esmaeelpour et al.
(17) found that there was a decreased choroidal thickness in diabetic patients with or without diabetic maculopathy. In contrast to our results, Kim et al. ${ }^{(4)}$ reported that the SFCT in DME eyes was significantly thicker than in non-DME eyes, Kase et al. ${ }^{(18)}$ also noted increased central choroidal thickness in eyes with DR compared with those without retinopathy. Other studies reported that SFCT increased with the severity of retinopathy ${ }^{(4,}$ 19).

The discrepancy between studies can be explained according to Nagaoka et al. ${ }^{(20)}$, who demonstrated impaired choroidal blood flow by laser Doppler flowmetry in diabetic eyes, which was further reduced in NPDR and even more in NPDR with DME. The authors hypothesized that hypoxia might initially thin the choroid with the resultant choroidal ischaemia that may disturb RPE pump function, and possibly contribute to the pathogenesis of DME. Subsequent production of vascular endothelial growth factor and increased blood flow might then account for the development of a thicker choroid with advancing retinopathy. On the other hand Ünsal et al. ${ }^{(11)}$ considered the decrease in choroidal thickness in DME to be false as a result of inhibition of the signal transduction and reflection from the choroid secondary to increased ocular opacity caused by macular edema. Vujosevic et al. ${ }^{(21)}$ stated that the choriocapillaris degeneration, and choroidal auto-infarction indirectly visualized as reduced choroidal thickness.

Our study revealed a weak negative correlation between SFCT and Log MAR in all groups, suggesting that if choroidal thickness is reduced, the vision will be affected. Other studies revealed a significant inverse correlation between SFCT and BCVA in the DME group ${ }^{(9,13)}$. This difference can be explained by different patient profiles as our study involve a wide variation in age in all groups 
(32- 68 years), and it was reported that choroidal thickness is negatively correlates with age ${ }^{(23)}$.

In our study, we also found a weak negative correlation between CMT and SFCT in diabetic groups (group II, group III). Also Regatieri et al. ${ }^{(5)}$ found a weak negative correlation in the DME group, whereas Lee et al. ${ }^{(15)}$ did not find any correlation. In another study, there was a statistically significant negative correlation between SFCT and CMT in DME group (22). This is differently from Eliwa et al. (9) who found a significant direct correlation between CMT and SFCT in the DME group.

In conclusion, a choroidal thinning appears to be a feature in diabetic subjects, a positive association between subfoveal choroidal thickness, macular oedema and vision was found. Spectral-domain OCT is a noninvasive technology to assess the choroid and may be a useful tool in the evaluation of chorioretinal vascular changes in DR.

\section{Financial support and sponsorship}

Nil.

\section{Conflicts of interest}

There are no conflicts of interest.

\section{REFERENCES}

1. Arevalo JF (2013): Diabetic macular edema: Current management. World J Diabetes, 4(6): 231-233.

2. Querques G, Lattanzio R, Querques L et al. (2012): Enhanced depth imaging optical coherence tomography in type 2 diabetes. Invest Ophthalmol Vis Sci., 60176024.

3. Mrejen S, Spaide RF (2013): Optical coherence tomography: Imaging of the choroid and beyond. Survey of ophthalmology, 58: 387-429.

4. Kim JT, Lee DH, Joe SG, Kim JG et al. (2013): Changes in choroidal thickness in relation to the severity of retinopathy and macular edema in type 2 diabetic patients. Invest Ophthalmol Vis Sci., 54(5): 3378-3384.

5. Regatieri CV, Branchini L, Carmody J et al. (2012): Choroidal thickness in patients with diabetic retinopathy analyzed by spectral-domain optical coherence tomography. Retina, 32(3): 563- 568.

6. Xu J, Xu L, Du K et al. (2013): Subfoveal choroidal thickness in diabetes and diabetic retinopathy. Ophthalmology, 120(10): 2023-2028.

7. Hannouche RZ, de Avila MP, Isaac DL et al. (2012): Correlation between central subfield thickness, visual acuity and structural changes in diabetic macular edema. Arq Bras Oftalmol., 7(3): 1590-1594.

8. Forooghian F, Stetson PF, Meyer SA et al. (2010): Relationship between photoreceptor outer segment length and visual acuity in diabetic macular edema. Retina, 30(1): 63-70.
9. Eliwa TF, Hegazy OS, Mahmoud SS et al. (2017): Choroidal Thickness Change in Patients with Diabetic Macular Edema. Ophthalmic Surg Lasers Imaging Retina, 48: 970-977.

10. Yiu G, Manjunath V, Chiu SJ et al. (2014): Effect of anti-vascular endothelial growth factor therapy on choroidal thickness in diabetic macular edema. Am J Ophthalmol., 158(4): 745-751.

11. Ünsal E, Eltutar K, Zirtiloğlu S et al. (2014). Choroidal thickness in patients with diabetic retinopathy. Clin Ophthalmol., 8: 637-642.

12. Sudhalkar A, Chhablani JK, Venkata A et al. (2015): Choroidal thickness in diabetic patients of indian ethnicity. Indian J Ophthalmol., 63(12): 912-916.

13. Wang S, Lin S, Zheng Y et al. (2015): Association of choroidal thickness with diabetic retinopathy at different stages. Zhonghua Yi Xue Za Zhi., 95(32): 2584-2588.

14. Galgauskas $S$, Laurinavičiūtė $G$, Norvydaitė $D$ et al. (2016): Changes in choroidal thickness and corneal parameters in diabetic eyes. Eur J Ophthalmol., 26(2): 163-167.

15. Lee HK, Lim JW, Shin MC et al. (2013): Comparison of choroidal thickness in patients with diabetes by spectral-domain optical coherence tomography. Korean J Ophthalmol., 27(6): 433-943.

16. Verma A, Nagpal $M$, Mehrotra $N$ et al. (2016): In vivo assessment of choroid in diabetic retinopathy by enhanced depth imaging in spectral do- main optical coherence tomography. Asia Pac J Ophthalmol (Phila), 5(5): 319-233.

17. Esmaeelpour M, Brunner S, Ansari-Shahrezaei $S$ et al. (2012): Choroidal thinning in diabetes type 1 detected by 3-dimensional 1060nm optical coherence tomography. Invest Ophthalmol Vis Sci., 53: 68036809.

18. Kase S, Endo H, Yokoi M et al. (2016).Choroidal thickness in diabetic retinopathy in relation to long-term systemic treatments for diabetes mellitus. Eur $J$ Ophthalmol., 26(2): 158-162.

19. Rewbury R, Want A, Varughese $R$ et al. (2016): Subfoveal choroidal thickness in patients with diabetic retinopathy and diabetic macular oedema. Eye (Lond), 30(12):1568-1572.

20. Nagaoka T, Kitaya N, Sugawara $R$ et al. (2004): Alteration of choroidal circulation in the foveal region in patients with type 2 diabetes. Br J Ophthalmol., 88(8): 1060-1063.

21. Vujosevic S, Martini F, Cavarzeran F et al. (2012). Macular and peripapillary choroidal thickness in diabetic patients. Retina, 32:1781-1790.

22. Hasan NA, Aldghaimy AH, Hamed MA et al. (2018): Correlation between choroidal thickness and central macular thickness measured by optical coherence tomography in nonproliferative diabetic retinopathy. Delta Journal of Ophthalmology, 19: 201-204.

23. Ohara Z, Tabuchi H, Nakakura $S$ et al. (2018): Changes in choroidal thickness in patients with diabetic retinopathy. Int Ophthalmol., 38: 279-286. 